\title{
Modulating Traffic Signal Phases to Realize Real-Time Traffic Control System
}

\author{
Rajendra S. Parmar ${ }^{1}$, Bhushan H. Trivedi ${ }^{2}$ \\ ${ }^{1}$ Gujarat Technology University (GTU), Ahmedabad, India \\ ${ }^{2}$ GLS Institute of Computer Technology, Ahmedabad, India \\ Email: raj@emerging-india.com, bhtrivedi@gmail.com
}

How to cite this paper: Parmar, R.S. and Trivedi, B.H. (2017) Modulating Traffic Signal Phases to Realize Real-Time Traffic Control System. Journal of Transportation Technologies, 7, 26-35.

http://dx.doi.org/10.4236/jtts.2017.71002

Received: October 14, 2016

Accepted: December 16, 2016

Published: December 19, 2016

Copyright ( 92017 by authors and Scientific Research Publishing Inc. This work is licensed under the Creative Commons Attribution International License (CC BY 4.0).

http://creativecommons.org/licenses/by/4.0/ cc) (7) Open Access

\begin{abstract}
This paper proposes innovations to address challenges emanating from road traffic congestion. Improving economies create more car owners resulting in increased automobile manufacturing, increased vehicle population giving rise to higher emission of $\mathrm{CO}_{2}$ resulting in traffic congestion. Congested traffic has idling vehicles which emit higher $\mathrm{CO}_{2}$ and pollution. Besides, traffic congestion increases turnaround time, delivery time, commuting time and related logistical aspects. Commuting time negatively impacts working hours. Unless the traffic congestion is mitigated, the economy will take a beating creating a vicious ecology cycle. Building new roads, bridges or reconditioning of infrastructure is not always the best possible solutions. Efficient traffic management is a key to country's economic growth. Various analytical models are employed to study, appreciate traffic congestion. The paper studies these models to infer that real time approach is the only solution. Several approaches are being worked on and few commercial systems too are available. These systems provide traffic information for course correction. However, it has latency and hence deviates from real time environment. Traffic congestion being highly dynamic in nature, it necessitates real time solution with real time inputs. It is proposed to integrate Real time traffic data with the traffic signal thus modulating the cycle timings at every junction. Deviation from static asymmetric cycle timing is implemented by assigning green phases based on density of vehicles. With minimalistic infrastructure and negligible incremental cost, the paper not only proposes to address traffic congestion but also paves the way for capturing traffic offenses, vehicle tracking and toll collection. The research is imminently realizable and makes a strong case for a PPP (Public Private Partnership) project.
\end{abstract}

\section{Keywords}

Vehicular Traffic, Vehicular Congestion, Detection System, Vehicular 
Congestion Detection System, Vehicle Mobility Sensors, Traffic Signals, Intelligent Traffic Signals, Dynamic Traffic Assignment

\section{Introduction}

The location accuracy from Global Positioning System (GPS) device is continuously improving; from 15 meters we have accuracy of 3 meters on systems that deploy WAAS (Wide Area Augmentation System). GPS uses at least three satellite data and communicates with GPS enabled receivers. WAAS uses a network of ground-based reference stations. Measurements from ground based stations along with GPS are sent to the geostationary WAAS satellites. WAAS satellites then sent the corrected data which is received by the WAAS enabled GPS devices.

PNDs (Personal Navigation Devices) are best suited for traffic application. The advantages are large displays, keyboards, arms length viewing and usability while driving. Besides, PND have room to accommodate speed, upcoming street, time of arrival and other parameters. However, in spite of these factors smart phones are preferred over PND. The mapping assistance is available through mapping giants like TeleNav, NavTeq (Navigation Technologies), Google, AT \& T. The PND based navigation systems is available at USD $\$ 70$ with a yearly update cost of USD $\$ 70$ approx whereas the smart phone besides the device cost \& data connectivity charges is available for USD $\$ 50$ for the navigation app. Ana$\log$ traffic receivers have an update frequency of 300 seconds. Garmin live traffic updates 1000 messages every 2 minutes on smart phones. Garmin devices with High Definition (HD) digital offer navigation services with live traffic updates for approx. more than USD $\$ 300$. The traffic update frequency is 30 seconds.

Motion X GS drive offers community contributed road alerts and reporting including accidents, hazards, police location, weather, and more. Tom-Tom provides traffic information by integrating variety of traditional data sources like governmental or third party data through road sensors or through mobile phone users. The system merges this information, analyses it and sends the data to users every three minutes.

In short, data come from millions of Garmin device owners, millions of cellular phone owners, incident reports, live information through radio feeds, news stations, NAVTEQ Traffic Supply and historical traffic data from each of these agencies. Some of the systems require downloading with periodic refreshing and work without the need for continuous connectivity.

\section{Related Work}

Proposed efficient Intelligent Traffic Control System (ITCS) system [1]. The digital-logic based principle being "a car can only move ahead if there is space for it" and "the signal remains green until the present cars have passed". Sensors are placed entry and exit of a road segment or a road leading to a traffic junction. 
These sensors provide information about the vehicles entering the junction and leaving the junction. This information is sufficient to know the number of vehicles on the road segment. Based on the vehicle population, the traffic signal phases are computed. The challenge here is fixed sensors. Fixed sensors have higher capital costs, installation cost and maintenance cost. It requires $24 \times 7$ power supply. Broadly speaking is prohibitively costly as all the traffic signals have to be equipped with the sensors.

Defines guidelines for Anthropocentric design for self-driving vehicles [2]. Presented intelligent Traffic Light Controllers (ITLC) based on microcontroller and microprocessor [3]. It has communication interface through which cycle times can be changed dynamically. Traffic signal related performance criterions can be logged and stored on a central server.

Google traffic displays traffic in real time by acquiring data from its users and calculating the speed [4]. On the map, Google shows green if traffic is moving along; yellow, if some traffic; red, if appreciable congestion.

GNSS-1 is the first generation system, which integrates existing GPS with other technologies. In USA, the satellite based component is the Wide Area Augmentation System (WAAS), in Europe it is the European Geostationary Navigation Overlay Service (EGNOS), and in Japan it is the Multi-Functional Satellite Augmentation System (MSAS). GNSS-2 is the second generation of system. These systems collate vehicle velocities. Commercial system like Tom Tom \& NavTeq capture PVT (Position, Velocity \& Time) to provide traffic information.

Academicians, researchers and corporations have been working on traffic control systems with a view to optimize the traffic signal phases, reduce signal idle time and address traffic congestion. The technology is based on ATCS (Adaptive Traffic Control System). The systems are evolving and continuously improvising to mitigate the traffic congestion problems. Before considering Adaptive Traffic Control Systems (ATCS) deployment, it is important to understand the reasons an ATCS is required.

\section{Contemporary Systems}

The software is based on Windows and OS and all of them have application software of their own. The traffic controllers used are Siemens M 50 series or 2070, Peek 3000E, Model 170, SITRAFFIC C8xx, C9xx, Econolite ASC/2 and NEMA AWA Delta $3 \mathrm{~N}$. The installation cost of ATCS per junction is approx. USD $\$ 65,000$.

SCOOT (Split Cycle Offset Optimization Technique) is a real time application for controlling traffic signals based on ATCS (Adaptive Traffic Control System) [5]. All traffic signals are connected and coordinated to allow smooth traffic flow. It deploys infrastructure information with the in-road sensors to compute traffic flow and traffic density. It offers all the information required to device vehicle routing thereby minimizing vehicle delays.

SCAT (Sydney Coordinated Adaptive Traffic System) is an ATCS using complex algorithms to generate traffic signal phases so as to minimize delays [6]. 
SCAT is a real time solution adjusting signal phases based on traffic area rather than vehicle count. The data is gathered through in-road loop sensors, which provides computing traffic density. SCAT in turn determines the duration of signal phases. SCAT provides operations and maintenance monitoring including; real time alarm monitoring, space and time diagrams as well as support from historical data which can help future planning.

Urban Traffic Optimization by Integrated Automation (UTOPIA) is integrated with SPOT to account to changes at the network level (Mauro and DiTaranto 1990). The parameters considered are vehicle detection, vehicle flow rate, traffic intensity, speed, road occupancy, density and type of vehicle. FHWA (Federal High-Way Administration), USA, developed ACS Lite which is widely deployable, at much reduced cost than traditional installation, operations and maintenance [7]. The system has CORSIM simulator and employs signal controllers from Eagle, Econolite, McCain and Peek controllers. The installations began around 2005. RHODES is a ATCS which began its implementations around 1990 [8]. RHODES predicts traffic levels taking sensors inputs from inductive loop sensors, RADAR, SONAR or video inputs. It then converts into lanes data and computes signal phases and communicates with server. RHODES also computes dynamic shortest real time to divert traffic from congested area to lean traffic area.

The comparison of the two systems with largest market share is tabulated in Table 1.

\section{Analytical Models}

Dynamic traffic assignment (DTA) is a subject of interest for the last decade and is yet maturing. The traffic networks are probabilistic and uncertain, various models were proposed. Broadly these models, model travelers' decision making, probabilistic travel time and random perception errors of individual travelers. Lighthill, Whitham, Richard introduced the continuum model (LWR model) based on fluid dynamics [9]. It proposes a function between traffic density and speed to capture the characteristics of traffic congestion formation. A congestion factor is evolved based on traffic data and principles of traffic congestion formation. The first-order continuum flow model, on dense traffic with equilibrium where $\mathrm{k}$ and $\mathrm{q}$ are density and flow respectively which depend on time $\mathrm{t}$ and position $x$

$$
\frac{d k(x, t)}{d t}+\frac{d q(x, t)}{d x}=0
$$

The model suggests preferred speed for vehicles. It is flawed, because, when passing is allowed, the preferred speed for each vehicle varies over time. Also the desired speeds among a group of vehicles vary.

PW Model: Payne-Whitham higher model introduces additional terms in the equation of motion proposed by LRW model, Navier-Stokes equation of motion for one-dimensional compressible flow with pressure and a relaxation term [9] [10]: 
Table 1. Comparison of contemporary Sysems.

\begin{tabular}{|c|c|c|c|}
\hline Parameters & SCOOT & SCAT & Rhodes \\
\hline Installations & 14 Countries; 200 systems & 27 Countries; 37,000 Intersections & \\
\hline Developer & $\begin{array}{l}\text { 1) IMTECH Traffic \& Infra UK Ltd } \\
\text { 2) Siemens Traffic Controls } \\
\text { 3) TRL Limited (Transport } \\
\text { Research Laboratory) funded by DfT } \\
\text { and the SCOOT suppliers }\end{array}$ & $\begin{array}{l}\text { 1) Australia } \\
\text { 2) New South Wales Government, } \\
\text { Australia }\end{array}$ & $\begin{array}{c}\text { Research team at University of } \\
\text { Arizona }\end{array}$ \\
\hline Year & Early Eighties; 1980 & 1975 & \\
\hline Description & $\begin{array}{l}\text { Split Cycle Offset Optimization } \\
\text { Technique }\end{array}$ & $\begin{array}{l}\text { Sydney Coordinated Adaptive Traffic } \\
\text { System }\end{array}$ & $\begin{array}{l}\text { Real-time Hierarchical } \\
\text { Optimizing Distributed } \\
\text { Effective System }\end{array}$ \\
\hline Type & On Line & On Line & Off Line \\
\hline Software & $\begin{array}{l}\text { 1) SCOOT Kernel, UTC software } \\
\text { 2) Microsoft Windows }\end{array}$ & $\begin{array}{l}\text { 1) Client Server Technology } \\
\text { 2) Windows OS }\end{array}$ & $\begin{array}{l}\text { Algorithm can be implemented } \\
\text { on any platform }\end{array}$ \\
\hline Performance & Reduces delay by $20 \%$ & Reduces delay by $5 \%$ to $45 \%$ & \\
\hline Detectors Technologies & On-Street Detectors; Inductive loops & On-Street Detectors; Inductive loops & $\begin{array}{l}\text { Inductive detectors, Video, } \\
\text { SONAR, RADAR }\end{array}$ \\
\hline Controllers & SIEMENS & $\begin{array}{l}\text { 1) SCAT compatible traffic } \\
\text { controller } \\
\text { 2) } 2070 \text { and } 170 \text { traffic signal } \\
\text { controllers }\end{array}$ & \\
\hline Deployment Cost & Very High & Very High & Very High \\
\hline Shortest Path & No & No & Yes \\
\hline $\begin{array}{l}\text { Comprehensive Traffic } \\
\text { Information }\end{array}$ & $\begin{array}{l}\text { ASTRID (Automatic SCOOT Traffic } \\
\text { Information Database) }\end{array}$ & Historical Data & No \\
\hline $\begin{array}{l}\text { Fault Monitoring \& } \\
\text { Management }\end{array}$ & Fault detection logic & Real Time Alarm System & Not available \\
\hline Features & $\begin{array}{l}\text { 1) Bus priority } \\
\text { 2) Manages pedestrians at junctions } \\
\text { 3) Estimate fuel emission } \\
\text { 4) INGRID (Integrated Incident } \\
\text { Detection) }\end{array}$ & $\begin{array}{l}\text { 1) Control air pollution } \\
\text { 2) Fuel consumption, delays and } \\
\text { reliability } \\
\text { 3) Real time alarm monitoring, space } \\
\text { and time diagrams }\end{array}$ & $\begin{array}{l}\text { 1) Real Time Dynamic shortest } \\
\text { path }\end{array}$ \\
\hline \multicolumn{4}{|c|}{$\begin{array}{l}\text { where } V \text { is traffic speed, } V e(k) \text { is the equilibrium speed-density relationship, } P(k) \\
\text { is traffic pressure, and } \lambda \text { is a coefficient. } \\
\text { Kalman filtering approach implements linear flow model [11]. Though here } \\
\text { too the observed density computed has inherent inaccuracies. Phillips' Model } \\
\text { incorporates mass conservation, momentum conservation, and energy conserva- } \\
\text { tion. Kuhnes' Model Incorporated sound speed and viscosity. Kerner and Kon- } \\
\text { hauser's model proposes that Initial homogeneous traffic flow can spontaneously } \\
\text { result in regions of high density and low average speed. These high-density re- } \\
\text { gions can move with or against the flow of traffic. Michalopoulos model does } \\
\text { not require the use of an equilibrium speed-density relationship. Zhang pro- } \\
\text { posed a non-equilibrium traffic flow model which is based on both empirical }\end{array}$} \\
\hline
\end{tabular}


evidence of traffic flow behavior and basic assumptions on drivers' reaction. Treiber Derived that in free flow, traffic information propagates downstream, while in congestion it travels upstream. Even though this method takes into account the way in which information propagates in traffic streams, it does not make use of any flow model [10] [11].

\section{Short Summary}

The study helps understand that (Table 2):

- Traffic congestion is spatial-temporal conglomeration of traffic volume in finite time and space,

- Traffic is multi-vehicle system with infinite equilibrium states (Not a unique equilibrium state),

- Vehicular traffic flow is nonlinear dynamic process,

- High-order models involve more complex partial differential equations, more variables which increases computational complexity and are more difficult to calibrate and implement,

- It is believed, high-order models, do not improve LWR model,

- Research till date has not been able to identify principles of traffic congestion OR conclusive conditions responsible for forming traffic congestion,

- Under the circumstances, congestion status of traffic flow can be evaluated in real time only [9].

\section{Proposition}

Traffic signal works on predetermined cycle times with each direction having green orange and red phases. These cycle times can be programmed to change or can be manually changed to cater to at peak and lean periods. However, a real time, dynamic modulation of traffic cycle time is desired to maximize the traffic

Table 2. Summary of related research work.

\begin{tabular}{|c|c|}
\hline Model & Limitations \\
\hline & $\begin{array}{l}\text { - } \quad \text { Model suggests Preferred speeds } \\
\text { - } \quad \text { Different vehicles have different preferred speeds }\end{array}$ \\
\hline $\begin{array}{l}\text { LRW (Lighthill, } \\
\text { Whitham, Richard) }\end{array}$ & $\begin{array}{l}\text { - Predicts abrupt speed change when a vehicle passes through a } \\
\text { shockwave, an action that is unrealistic in real world. }\end{array}$ \\
\hline OR Continuum Model & $\begin{array}{l}\text { - LWR model fails to predict instabilities of stop-start traffic } \\
\text { - LWR model assumes zero reaction time which does not happen } \\
\text { in real world }\end{array}$ \\
\hline $\begin{array}{l}\text { PW Model: } \\
\text { Payne-Whitham }\end{array}$ & $\begin{array}{l}\text { - Like LRW model, It does not remove all the shock waves } \\
\text { - Vehicles are expected to adjust their speeds in relation to the rear } \\
\text { vehicles, while in reality vehicles respond to thread } \\
\text { - Proposed wave speeds are greater than traffic speeds. Again } \\
\text { primarily because it derives the recommendations from rear vehicles }\end{array}$ \\
\hline $\begin{array}{l}\text { Kalman filtering } \\
\text { approach }\end{array}$ & $\begin{array}{l}\text { - Velocity of an individual vehicle is assumed to correspond to the } \\
\text { velocity at that given location } \\
\text { - The velocity-to-density conversion, introduces error } \\
\text { - Measurement error in the velocity }\end{array}$ \\
\hline
\end{tabular}


flow. Has demonstrated need to utilize unused green phases [12]. It is proposed to assigning green phases proportional to the vehicular density in the respective direction. The strategy translates into uniform dispersion of vehicles rather than uniform cycle time to each direction. This will result in decongesting congested vehicles and evenly distributing vehicular densities.

The important parameters to compute are listed in Table 3.

The paper integrates vehicle registration, mapping application, traffic monitoring and modulating the traffic signals (Table 3 ).

\subsection{Vehicle Mapping}

Vehicle mapping assigns a device ID to a vehicle. Extreme care is taken to maintain users' privacy and data confidentiality. Besides, vehicle manufacturer, model, vehicle area (length, breadth), fuel type, fuel economy and other parameters of importance are logged. The make and model, fuel type, distance travelled are parameters for computing and monitoring pollution data from the vehicular emission. The time stamped data with latitude \& longitude, road name builds a history, behavior and knowledge database about the vehicle, road segment. The ID also identifies vehicles as emergency vehicle, transport vehicle, commercial, public vehicle or private vehicle.

\subsection{Mapping Application}

Mapping application is intended to capture infrastructure characteristics like length of road segment, number of lanes that includes width of road segment, right of travel direction-unidirectional or bidirectional and quality of road segment to decide average speed of vehicle on the road segment. The application computes how many vehicles can be present in the road segment. Besides it logs the latitude and longitude of the ends of a road segment, name of the road segment which enables the graphic application to render the map. A survey vehicle is equipped with the infrastructure information capturing application drives through the entire city with a data entry team. The application prompts for nomenclature (Location description) every time there is a change in lat/long. The average speed is captured from the length of the road segment and time taken to traverse the same. The speed is related to the quality of road segment. The user enters number of lanes, right of direction. The user also enters the number of converging roads and traffic signals at both ends of the road segment.

Table 3. Traffic junction parameters of importance.

\begin{tabular}{ccc}
\hline Parameter & Value & Unit \\
\hline Min. Green Phase Time & $\mathrm{t}_{\min }$ & $\mathrm{s}$ \\
Max. Green phase time & $\mathrm{t}_{\max }$ & $\mathrm{s}$ \\
Time to cross junction & $\mathrm{t}_{\mathrm{t}}$ & $\mathrm{s}$ \\
Vehicles at junction & $\mathrm{v} 1$ & No \\
Capacity to hold Vehicles & $\mathrm{cl}$ & No \\
Vehicles at next junction & $\mathrm{v} 2$ & No \\
Capacity to hold Vehicles & $\mathrm{c} 2$ & No \\
\hline
\end{tabular}




\subsection{Traffic Monitoring}

The application periodically captures lat/long. The periodicity is limited to remove data redundancy and only create useful data, rendering data compression. The vehicle position is updated in the server. The speed computation is done by taking two reasonable samples of positions with a time stamp. The sample provides distance and time to travel the distance, thus giving average speed. Vehicle density is computed by adding up all the vehicles whose position falls between the lat/long of two junctions. Infrastructure details are obtained from the database detailing allowable speeds on the road segment, length of the road segment and the capacity of the road segment besides other parameters. Congestion index is computed by comparing the present vehicular density to the capacity of the road segment. Congestion index of 0.8 to 1.0 means heavy congestion, if the index is between 0.6 to 0.8 it would mean moderate traffic and below 0.6 is free flowing traffic. The server responds with all the relevant information to assist the driver.

\subsection{Modulating the Traffic Signals}

Traffic is controlled intelligently after accurate, real time data is obtained. So far all the directions were assigned fair, equal signal phases. However, many a times when there is no contest or contention for a direction because there are no vehicles. Hence asymmetric signal phase is proposed. The proposal is to give priority to a direction with maximum number of waiting vehicles. However, there is a possibility of starvation amongst directions with fewer vehicles. Hence waiting time is incorporated along with number of vehicles. Waiting time is summation of waiting time of all vehicles at a junction. The intension is to normalize waiting time of vehicles. This will ensure a truly fair assignment of signaling to different directions. The central server has data of all the vehicles on the road which includes the road segment they are in, average speeds and vehicles waiting at a traffic junction besides other relevant information.

1) The algorithm is designed to service the direction with largest number of vehicles waiting for the longest time expressed as:

$$
\tau=\sum_{1}^{N}\left(\int_{T 1}^{T 2} d t\right)
$$

$\tau=$ Cumulative waiting time (CWT) of vehicles for a direction; $T 1=$ Entry time of vehicle; $T 2=$ Time at computation; $\mathrm{N}=$ Number of vehicles.

The algorithm continuously evaluates green phase and assigns to a direction. While doing so, enough care is taken not to starve the other directions indefinitely. The upper and lower bound of green \& red phases ensures that the direction with dense vehicle population is assigned a red phase and the sparsely dense direction too gets its share of green phase.

2) The green phase is assigned only when the road segment ahead has capacity to accommodate additional vehicles OR the green phase timing is defined based on how many vehicles it can accommodate. 
3) There might be a case when all the signal phases are red indicating heavy congestion. It allows the road ahead to create enough buffer to accommodate additional vehicles. This ensures the system allows the congestion to build up to a manageable level and not frustrating level of congestion.

\section{Future Work Proposed}

[13] demonstrated that violations and offenses like speeding, license plate cloning, vehicular robbery, driving with influence of alcohol can be detected. The proposed system in this paper detects over speeding vehicles. The device installed on the vehicle, has a unique ID. Addressable vehicles are monitored for violations like over speeding, jumping traffic signals, wrong parking or long parking hours. An over-speeding vehicle is detected immediately and so is the detection of a breakdown. Needless to say, the toll collection is automated. The system collets toll based on travel zones. With exact vehicle location, vehicle speed, speed limit of the road segment and other parameters being available, post accident analyses becomes a trivial matter. Besides the routine display parameters, the dash board can indicate distances of neighboring vehicles, their speeds, cross roads ahead with a notification of green, red or orange situation. Lastly, real time traffic control system can also pave the way to eliminate signaling systems at every traffic junction by implementing signaling indications in each vehicle dash board directly. This will make the traffic signaling systems redundant, resulting in large savings in its capital as well as operational \& recurring maintenance costs.

\section{References}

[1] Bilal, J.M. and Jacob, D. (2007) Intelligent Traffic Control System. IEEE International Conference on Signal Processing and Communications (ICSPC 2007), Dubai, 24-27 November 2007.

[2] Mladenovic, M.N., Abbas, M. and McPherson, T. (2014) Development of Socially Sustainable Traffic-Control Principles for Self-Driving Vehicles: The Ethics of Anthropocentric Design. IEEE International Symposium on Ethics in Science, Technology and Engineering.

[3] Chavan, S.S., Deshpande, R.S. and Rana, J.G. (2009) Design of Intelligent Traffic Light Controller Using Embedded System. Second International Conference on Emerging Trends in Engineering and Technology, ICETET.

[4] http://www.theconnectivist.com/2013/07/how-google-tracks-traffic/

[5] http://www.scoot-utc.com/

[6] http://www.scats.com.au/

[7] Shelby, S.G., Bullock, D.M., Gettman, D., Ghaman, R.S., Sabra, Z.A. and Soyke, N. (2008) An Overview and Performance Evaluation of ACS Lite-A Low Cost Adaptive Signal Control System. 87th TRB Annual Meeting, Washington DC, January 2008.

[8] RHODES—Real Time Hierarchical Optimized Distributed Effective System. http://ocw.nctu.edu.tw/course/sc011/2012-08-23-1.pdf

[9] Zhang, W., Tan, G.Z., Ding, N. and Wang, G.Y. (2012) Traffic Congestion Evaluation and Signal Control Optimization Based on Wireless Sensor Networks: Model 
and Algorithms. School of Computer Science and Technology, Dalian University of Technology, Dalian.

[10] http://people.umass.edu/ndh/TFT/Ch15\%20High.pdf

[11] Herrera, J.C. and Bayen, A.M. Departamento de Ingeniería de Transporte y Logística, Pontificia Universidad Católica de Chile, Chile, Systems Engineering, Department of Civil and Environmental Engineering, University of California, Berkeley, United States; Incorporation of Lagrangian Measurements in Freeway Traffic State Estimation.

[12] Parmar, R.S. and Trivedi, B. (2014) Real Time Computation of Optimal Signal Timing to Maximize Vehicular Throughput for a Traffic Junction. 3rd International Conference on Eco-Friendly Computing and Communication Systems (ICECCS), ICECCS 2014, Mangalore, 18-21 December 2014.

[13] Ou, G.Y., Gao, Y. and Liu, Y. (2012) Real-Time Vehicular Traffic Violation Detection in Traffic Monitoring Stream. IEEE/WIC/ACM International Conferences on Web Intelligence and Intelligent Agent Technology.

\section{Submit or recommend next manuscript to SCIRP and we will provide best} service for you:

Accepting pre-submission inquiries through Email, Facebook, LinkedIn, Twitter, etc. A wide selection of journals (inclusive of 9 subjects, more than 200 journals)

Providing 24-hour high-quality service

User-friendly online submission system

Fair and swift peer-review system

Efficient typesetting and proofreading procedure

Display of the result of downloads and visits, as well as the number of cited articles

Maximum dissemination of your research work

Submit your manuscript at: http://papersubmission.scirp.org/

Orcontact jtts@scirp.org 